\title{
Generic properties of Mañé's set of exact magnetic Lagrangians
}

\author{
Alexandre Rocha
}

December 17, 2019

\begin{abstract}
Let $M$ be a closed manifold and $L$ an exact magnetic Lagrangian. In this paper we proved that there exists a residual $\mathcal{G}$ of $H^{1}(M ; \mathbb{R})$ such that the property:

$$
\widetilde{\mathcal{M}}(c)=\widetilde{\mathcal{A}}(c)=\widetilde{\mathcal{N}}(c), \forall c \in \mathcal{G}
$$

with $\widetilde{\mathcal{M}}(c)$ supports on a uniquely ergodic measure, is generic in the family of exact magnetic Lagrangians.

We also prove that, fixed the cohomology class $c$, there exists a residual set of exact magnetic Lagrangians such that when this unique measure is supported on a periodic orbit, this orbit is hyperbolic and its stable and unstable manifolds intersect transversally. This result is a version of Theorem D of [4] for the exact magnetic Lagrangian case.
\end{abstract}

\section{Introduction}

Let $M$ be a closed manifold equipped with a Riemannian metric $g=\langle.,$.$\rangle . A$ Lagrangian $L: T M \rightarrow \mathbb{R}$ is called exact magnetic Lagrangian if

$$
L(x, v)=\frac{\|v\|^{2}}{2}+\eta_{x}(v)
$$

for some non-closed 1-form $\eta_{x}$. The Euler-Lagrangian flow of this Lagrangian can also be obtained as magnetic flow associated to an exact 2-form $\Omega=-d \eta_{x}$.

This type of Lagrangian fits into Mather's theory, as developed by R. Mañé and A. Fathi, about Tonelli Lagrangians, namely, it is fiberwise convex and superlinear. 
Let $\mathfrak{M}(L)$ be the set of action minimizing measures. Recall that $\mathfrak{M}(L)$ is the set of $\mu$ Borel probability measures on $T M$ which are invariant under the Euler-Lagrange flow $\varphi_{t}$ generated by $L$ and minimizes the action.

Since the Euler Lagrange flow generated by $L$ does not change by adding a closed one form $\zeta$, we also consider the action minimizing measures $\mathfrak{M}(L-\zeta)$. The minimal action value, depends only on the cohomology class $c=[\zeta] \in H^{1}(M, \mathbb{R})$ of the closed one form, so it is denoted by $-\alpha(c)$. It is known that $\alpha(c)$ is the energy level that contains the Mather set for the cohomology class c:

$$
\widetilde{\mathcal{M}}_{c}(L)=\overline{\bigcup_{\mu \in \mathfrak{M}(L-\zeta)} \operatorname{supp}(\mu)} .
$$

$\widetilde{\mathcal{M}}_{c}(L)$ is a compact invariant set which is a graph over a compact subset $\mathcal{M}_{c}(L)$ of $M$, the projected Mather set (see [10]). $\mathcal{M}_{c}(L)$ is laminated by curves, which are global (or time independent) minimizers. Mather also proved that the function $c \mapsto \alpha(c)$ is convex and superlinear.

In general, $\widetilde{\mathcal{M}}_{c}(L)$ is contained in another compact invariant set, which is also a graph whose projection is laminated by global minimizers: the Aubry set for the cohomology class $c$, denoted by $\widetilde{\mathcal{A}}_{c}(L)$.

In order to state our results, we need to introduce the Aubry set and the Mañé set for a general Tonelli Lagrangian:

Let $\xi$ be a closed one form representative of the cohomology class $c=[\xi]$. The action of a $C^{1}$ curve $\gamma:[0, T] \rightarrow M$ is defined by

$$
A_{c}^{L+k}(\gamma)=\int_{0}^{T}[L(\gamma, \dot{\gamma})-\xi(\gamma)(\dot{\gamma})+k] d t
$$

where $k$ is a real number. The energy level $c(L-\xi)$, namely Mañés critical value of the Lagrangian $L-c$, which depends only on the cohomology class $c=[\xi]$, may be characterized in several ways. $c(L-\xi)$ is defined by Mañé as the infimum of the numbers $k$ such that the action $A_{c}^{L+k}(\delta)$ is nonnegative for every closed curve $\delta:[0, T] \rightarrow M$.

Recall that, for a given real number $k$ the action potential $\Phi_{c}^{L+k}: M \times M \rightarrow \mathbb{R}$ is defined by

$$
\Phi_{c}^{L+k}(x, y)=\inf A_{c}^{L+k}(\gamma)
$$

infimum taken over the curves $\gamma$ joining $x$ the $y$.

Mañé proved that $c(L-\xi)=-\alpha([\xi])=-\alpha(c)$, and that $\alpha(c)$ is the smallest number such that the action potential is finite. In other words, if $k<\alpha(c)$, then 
$\Phi_{L-\xi+k}(x, y)=-\infty$ and if $k \geq \alpha(c), \Phi_{L-\xi+k}(x, y) \in \mathbb{R}$. We introduce the following notations: $\Phi_{c}^{L}:=\Phi_{c}^{L+\alpha(c)}$ and $A_{c}^{L}:=A_{c}^{L+\alpha(c)}$.

Observe that by Tonelli's Therorem (See for example in [5]), for fixed $t>0$, there always exists a minimizing extremal curve connecting $x$ to $y$ in time $t$. The potential calculates the global (or time independent) infimum of the action. This value may not be realized by a curve.

The potential $\Phi_{c}^{L}$ is not symmetric in general but

$$
\delta_{M}(x, y)=\Phi_{c}^{L}(x, y)+\Phi_{c}^{L}(y, x)
$$

is a pseudo-metric. A curve $\gamma: \mathbb{R} \rightarrow M$ is called semistatic if minimizes action between any of its points:

$$
A_{c}^{L}\left(\left.\gamma\right|_{[a, b]}\right)=\Phi_{c}^{L}(\gamma(a), \gamma(b))
$$

and $\gamma$ is called static if is semistatic and $\delta_{M}(\gamma(a), \gamma(b))=0$ for any $a, b \in \mathbb{R}$.

Actually, the orbits contained in the Mather set $\widetilde{\mathcal{M}}_{c}(L)$ project onto static curves. The Mañé set $\widetilde{\mathcal{N}}_{c}(L)$ is the set of the points $(x, v) \in T M$ such that the projection $\gamma(t)=\pi \circ \varphi_{t}(x, v)$ is a semistatic curve and the Aubry set $\widetilde{\mathcal{A}}_{c}(L)$ is the set of the points $(x, v) \in T M$ such that the projection $\gamma(t)=\pi \circ \varphi_{t}(x, v)$ is a static curve.

Mañé proved that $\widetilde{\mathcal{A}}_{c}(L)$ is chain recurrent and it is a challenging question to describe the dynamics of the Euler-Lagrange flow restricted to $\widetilde{\mathcal{A}}_{c}(L)$. All these properties are proven in [5]. We introduce the following notations: $\mathcal{A}_{c}(L)$ and $\mathcal{N}_{c}(L)$ to represent the projected Aubry and Mañé sets of Lagrangian $L-c$, respectively.

The notion of genericity in the context of Lagrangian systems is provided by Mañé in [8]. The idea is to make special perturbations by adding a potential: $L(x, v)+\Psi(x)$, for $\Psi \in C^{\infty}(M)$. A property is generic in the sense of Mañé if it is valid for every Lagrangians $L(x, v)+\Phi(x)$ with $\Phi$ contained in a residual subset $\mathcal{O}$. In this sense, G. Contreras and G. Paternain, prove in [6] (Theorem C) that for a fixed cohomology class $c$, the property

$$
\widetilde{\mathcal{M}}_{c}(L)=\widetilde{\mathcal{A}}_{c}(L)=\widetilde{\mathcal{N}}_{c}(L),
$$

with $\widetilde{\mathcal{M}}_{c}(L)$ supports a uniquely ergodic measure is generic. Furthermore, J. Zhang proves in 12 that for generic Tonelli Lagrangian, there exists a residual set $\mathcal{G} \subset H^{1}(M ; \mathbb{R})$ such that 1 holds for any $c \in \mathcal{G}$.

In general, when we are dealing with a specific class of Lagrangians, perturbations by adding a potential are not allowed. However the main goal of this paper is to prove some generic properties of minimizing sets for the family of exact magnetic Lagrangians. 
In the work of Miranda, J. A. G [7], the perturbations of a magnetic flow are made on the space of 2 -forms on $M$ with the $C^{\infty}$ topology that preserve the cohomology class (in particular on the exact 2-forms). He proves a version of the Kupka-Smale Theorem for this class of flows, when $M$ is a surface. More recently, Arbieto, A. and Castro, F. [1] generalize for any dimension of $M$.

Let us take $\Gamma^{\infty}(M)$ the set of smooth 1-forms on $M$ endowed with the metric

$$
d\left(\omega_{1}, \omega_{2}\right)=\sum_{k \in \mathbb{N}} \frac{\arctan \left(\left\|\omega_{1}-\omega_{2}\right\|_{k}\right)}{2^{k}},
$$

denoting by $\|\omega\|_{k}$ the $C^{k}$-norm of the 1 -form $\omega$, that is: given a $C^{\infty} 1$-form $\omega$, we associate a $C^{\infty}$ field $X$ on $M$ such that $\omega_{x}(v)=\langle X(x), v\rangle$. We define the $C^{k}$-norm $\|\omega\|_{k}$ of $\omega$ as the $C^{k}$-norm of $X$.

The a first integral of the flow $\varphi_{t}$ is the energy function $E: T M \rightarrow \mathbb{R}$, defined by

$$
E(x, v)=\frac{\partial L}{\partial v}(x, v)(v)-L(x, v) .
$$

Recall that

$$
e_{0}=-\min _{x \in M} L(x, 0)=\max _{x \in M} E(x, 0) .
$$

We have $\min \alpha \geq e_{0}$ and for any $k>e_{0}$, the energy level $E^{-1}(k)$ is a hypersurface of $T M$. Let state our main result.

Theorem 1 For an exact magnetic Lagrangian $L$ defined on $T M$, there exist a residual set $\mathcal{G} \subset H^{1}(M ; \mathbb{R})$ and a residual $\mathcal{O} \subset \Gamma^{\infty}(M)$ such that

$$
\widetilde{\mathcal{M}}_{c}(L+\varphi)=\widetilde{\mathcal{A}}_{c}(L+\varphi)=\widetilde{\mathcal{N}}_{c}(L+\varphi), \forall c \in \mathcal{G}, \forall \varphi \in \mathcal{O}
$$

with $\widetilde{\mathcal{M}}_{c}(L+\varphi)$ supports a uniquely ergodic measure.

Actually, before we will prove the following proposition which allows us to perturb Tonelli Lagrangians by adding 1-forms.

Proposition 2 Let $L$ be a Tonelli Lagrangian and c be a cohomology class with $\alpha(c)>$ $e_{0}$. If $\widetilde{\mathcal{M}}_{c}(L)=\operatorname{supp}\left(\mu_{0}\right)$ supports on a uniquely ergodic measure $\mu_{0}$, then there exists a $C^{\infty}$ 1-form $\eta$ (sufficiently close to zero) such that

$$
\widetilde{\mathcal{M}}_{c}(L+\eta)=\widetilde{\mathcal{A}}_{c}(L+\eta)=\widetilde{\mathcal{N}}_{c}(L+\eta)=\widetilde{\mathcal{M}}_{c}(L) .
$$

In Section 3 we shall present a version for the family of exact magnetic Lagrangians of a theorem stated in [9] by Mañé, proved in ([4, Theorem D). 
Theorem 3 Let $L$ be an exact magnetic Lagrangian. Then there exists a residual set $\mathcal{O}$ of $\Gamma^{\infty}(M)$ such that for every $\omega \in \mathcal{O}$ the Lagrangian $L+\omega$ has a unique minimizing measure, uniquely ergodic $\mu_{\omega}$. Moreover, every periodic orbit $\Gamma$ which is the support of $\mu_{\omega}$, for some $\omega \in \mathcal{O}$, is hyperbolic and its stable and unstable manifolds intersect transversally $W^{s}(\Gamma) \pitchfork W^{u}(\Gamma)$.

\section{Perturbation of a Tonelli Lagrangian by adding a 1-form}

In this section, we will dedicate to prove Proposition 2 and Theorem 1. In order to prove the results we need of the following lemma:

Lemma 4 Let $L: T M \rightarrow \mathbb{R}$ be a Tonelli Lagrangian and $c$ be a cohomology class with $\alpha(c)>e_{0}$. Then there exist a neighborhood $\mathcal{U} \subset T M$ of $\widetilde{\mathcal{A}}(c)$, a $C^{\infty}$ vector field $X: M \rightarrow T M$ and $K>0$ such that for every $(x, v) \in \mathcal{U}$,

$$
\left(\frac{\partial L}{\partial v}(x, X(x))-\frac{\partial L}{\partial v}(x, 0)\right)(v) \geq K>0 .
$$

Proof: Since $\left(\left.\pi\right|_{\mathcal{A}(c)}\right)^{-1}$ is a Lipschitz map we can consider $\xi: M \rightarrow T M$ a Lipschitz extension of $\left(\left.\pi\right|_{\mathcal{A}(c)}\right)^{-1}$ to $M$. We define a Lipschitz map $F: T M \rightarrow \mathbb{R}$ by $F(x, v)=$ $\left(\frac{\partial L}{\partial v}(x, \xi(x))-\frac{\partial L}{\partial v}(x, 0)\right)(v)$. Let us prove first that $L_{0}(x, v)=L(x, v)-\frac{\partial L}{\partial v}(x, 0)(v)-$ $L(x, 0) \geq 0$. Indeed, observe that $L_{0}$ is a convex superlinear function, $L_{0}(x, 0)=0$ and $\frac{\partial L_{0}}{\partial v}(x, 0)=0$. Therefore $L_{0}(x, \star)$ has its minimum at $v=0$, hence $L_{0}(x, v) \geq 0$. Now let us take $(x, v) \in \widetilde{\mathcal{A}}(c)$, i.e. $v=\xi(x)$. Then

$$
\begin{aligned}
F(x, v) & =\left(\frac{\partial L}{\partial v}(x, \xi(x))-\frac{\partial L}{\partial v}(x, 0)\right)(\xi(x)) \\
& =\frac{\partial L}{\partial v}(x, \xi(x))(\xi(x))-L(x, \xi(x))+L(x, \xi(x))-\frac{\partial L}{\partial v}(x, 0)(\xi(x)) \\
& =E(x, \xi(x))+\left[L(x, \xi(x))-\frac{\partial L}{\partial v}(x, 0)(\xi(x))-L(x, 0)\right]+L(x, 0) \\
& =\alpha(c)+L_{0}(x, \xi(x))+L(x, 0) \geq \alpha(c)-e_{0}>0,
\end{aligned}
$$

because $e_{0}=-\min _{x \in M} L(x, 0)$.

By the continuity of $F$ we obtain a neighborhood $\mathcal{U} \subset T M$ of $\widetilde{\mathcal{A}}(c)$ such that $\left.F\right|_{\mathcal{U}}>\frac{c_{0}-e_{0}}{2}>0$. Since $\widetilde{\mathcal{A}}(c) \subset\{(x, v) \in T M:\|v\| \leq B\}$ for some $B>0$, we can suppose that $\mathcal{U} \subset\{(x, v) \in T M:\|v\|<D\}$ for some $D>0$. By Whitney's approximation 
Theorem, given $\delta>0$ there exists a $C^{\infty}$ map $X: M \rightarrow T M$ with

$$
\|X(x)-\xi(x)\|<\delta, \forall x \in M
$$

It follows from continuity of $L_{v}$ taking $\delta>0$ smaller if necessary, we have

$$
\left\|\frac{\partial L}{\partial v}(x, X(x))-\frac{\partial L}{\partial v}(x, \xi(x))\right\|<\frac{\alpha(c)-e_{0}}{4 D}, \forall x \in M .
$$

Therefore, if $(x, v) \in \mathcal{U}$,

$$
\begin{aligned}
\left|F(x, v)-\left(\frac{\partial L}{\partial v}(x, X(x))-\frac{\partial L}{\partial v}(x, 0)\right)(v)\right| & =\left|\left(\frac{\partial L}{\partial v}(x, \xi(x))-\frac{\partial L}{\partial v}(x, X(x))\right)(v)\right| \\
& \leq\left\|\frac{\partial L}{\partial v}(x, X(x))-\frac{\partial L}{\partial v}(x, \xi(x))\right\|\|v\| \\
& <\frac{\alpha(c)-e_{0}}{4 D} \cdot D=\frac{\alpha(c)-e_{0}}{4} .
\end{aligned}
$$

and

$$
\left(\frac{\partial L}{\partial v}(x, X(x))-\frac{\partial L}{\partial v}(x, 0)\right) v>F(x, v)-\frac{\alpha(c)-e_{0}}{4}>\frac{\alpha(c)-e_{0}}{4} \stackrel{\text { def }}{=} K>0 .
$$

The next step is to show the upper-semicontinuity of the Mañé set for Tonelli Lagranginas when we add a 1-form:

Proposition 5 Let $L: T M \rightarrow \mathbb{R}$ be a Tonelli Lagrangian. As a set-valued function, $(\xi, c) \in \Gamma^{\infty}(M) \times H^{1}(M ; \mathbb{R}) \longmapsto \widetilde{\mathcal{N}}_{c}(L+\xi)$ is upper-semicontinuous, that is given a neighborhood $\mathcal{V}$ of $\widetilde{\mathcal{N}}_{c_{0}}\left(L+\xi_{0}\right)$ in $T M$ there exists a neighborhood $U \times V$ of $\left(\xi_{0}, c_{0}\right)$ in $\Gamma^{\infty}(M) \times H^{1}(M ; \mathbb{R})$ such that $\widetilde{\mathcal{N}}_{c}(L+\xi) \subset \mathcal{V}$ for every $(\xi, c) \in U \times V$.

Before proving this proposition, we shall prove the Mather's $\alpha$-function depends continuously of $(\xi, c) \in \Gamma^{\infty}(M) \times H^{1}(M ; \mathbb{R})$. We will prove that it holds for any Tonelli Lagrangians.

Lemma 6 Let $L: T M \rightarrow \mathbb{R}$ be a Tonelli Lagrangian. The map $(\xi, \lambda) \in \Gamma^{\infty}(M) \times$ $H^{1}(M ; \mathbb{R}) \longmapsto \alpha_{L+\xi}(\lambda)=c(L+\xi-\lambda)$ is continuous.

Proof: Suppose that $\left(\xi_{n}, \lambda_{n}\right) \rightarrow(\xi, \lambda), \alpha_{n}=\alpha_{L+\xi_{n}}\left(\lambda_{n}\right)$, and $\alpha=\alpha_{L+\xi}(\lambda)$. We shall prove that $\alpha_{n} \rightarrow \alpha$. Let us take $\xi_{n}-\lambda_{n}=\sigma_{n}$ and $\xi-\lambda=\sigma$. By the duality there exist vector fields $X_{n}$ and $X$ on $M$ such that

$$
\sigma_{n}(x)(v)=\left\langle X_{n}(x), v\right\rangle \text { and } \sigma(x)(v)=\langle X(x), v\rangle .
$$


Observe that the energy function $E_{n}$ of $L+\sigma_{n}$ is

$$
E_{n}(x, v)=\left(L(x, v)+\left\langle X_{n}(x), v\right\rangle\right)_{v} v-\left(L+\left\langle X_{n}(x), v\right\rangle\right)=L_{v} v-L=E(x, v),
$$

for every $n$, where $E$ is the energy function of $L+\sigma$. It is know that $E$ is a superlinear function. Then there exist $B>0$ such that $E(x, v) \geq\|v\|-B$ for every $(x, v) \in T M$.

Observe that $-\alpha_{n}=\int_{T M}\left(L(x, v)+\sigma_{n}\right) d \mu_{n}$ where $\mu_{n}$ is a minimizing measure $\mu_{n}$ of $L+\sigma_{n}$. Thus $\alpha_{n}=E_{n}\left(\operatorname{supp}\left(\mu_{n}\right)\right)=E\left(\operatorname{supp}\left(\mu_{n}\right)\right)$.

Since $\left(\xi_{n}, \lambda_{n}\right) \rightarrow(\xi, \lambda)$, given $\varepsilon>0$, there exists $n_{0} \in \mathbb{N}$ such that

$$
n>n_{0} \Rightarrow\left\|X_{n}(x)-X(x)\right\|<\varepsilon, \forall x \in M
$$

Moreover, we have that $-\alpha \leq \int_{T M}(L+\sigma) d \mu_{n}$. Thus, for every $n>n_{0}$, we obtain

$$
\begin{aligned}
\alpha_{n}-\alpha & \leq-\int_{T M}\left(L+\sigma_{n}\right) d \mu_{n}+\int_{T M}(L+\sigma) d \mu_{n} \\
& =\int_{T M}\left\langle X_{n}(x), v\right\rangle-\langle X(x), v\rangle d \mu_{n} \\
& \leq \int_{T M}\left\|X_{n}(x)-X(x)\right\|\|v\| d \mu_{n}<\int_{T M} \varepsilon\|v\| d \mu_{n} \\
& \leq \varepsilon \int_{T M}(E(x, v)+B) d \mu_{n}=\varepsilon \alpha_{n}+\varepsilon B .
\end{aligned}
$$

Taking $\varepsilon=\frac{1}{2}$ above, we conclude

$$
0<\alpha_{n} \leq \frac{\alpha+\varepsilon B}{1-\varepsilon}=2 \alpha+B
$$

Given $\bar{\varepsilon}>0$, we take $\varepsilon=\min \left\{\frac{\bar{\varepsilon}}{2 \alpha+2 B}, \frac{1}{2}\right\}>0$ in (3). So we can use (44) to obtain that there exists $n_{1} \in \mathbb{N}$ such that $\alpha_{n}-\alpha \leq \varepsilon \alpha_{n}+\varepsilon B \leq \bar{\varepsilon}$ for every $n>n_{1}$.

There exists $n_{2} \in \mathbb{N}$ such that

$$
n>n_{2} \Rightarrow\left\|X_{n}(x)-X(x)\right\|<\frac{\bar{\varepsilon}}{\alpha+B}, \forall x \in M .
$$

Let $\mu$ be a minimizing measure of $L+\sigma$. Then

$$
\begin{aligned}
\alpha-\alpha_{n} & \leq-\int_{T M}(L+\sigma) d \mu+\int_{T M}\left(L+\sigma_{n}\right) d \mu \\
& \leq \int_{T M}\left\|X_{n}(x)-X(x)\right\|\|v\| d \mu \\
& \leq \int_{T M} \frac{\bar{\varepsilon}}{\alpha+B}\|v\| d \mu \leq \frac{\bar{\varepsilon}}{\alpha+B} \int_{T M}(E(x, v)+B) d \mu=\bar{\varepsilon}
\end{aligned}
$$

Therefore, if $n>\max \left\{n_{0}, n_{1}, n_{2}\right\}$, we have $\left|\alpha-\alpha_{n}\right|<\bar{\varepsilon}$. 
Proof: (of Proposition 5) Let $\left(\xi_{0}, c_{0}\right)$ be a point in $\Gamma^{\infty}(M) \times H^{1}(M ; \mathbb{R})$. Since the Mañé set is contained in the energy level $\widetilde{\mathcal{N}}_{c}(L) \subset E^{-1}(\alpha(c))$, it follows from previous lemma that there exist a neighborhood $U \times V$ of $\left(\xi_{0}, c_{0}\right)$ in $\Gamma^{\infty}(M) \times H^{1}(M ; \mathbb{R})$ and a compact subset $\mathcal{K} \subset T M$ such that $\widetilde{\mathcal{N}}_{c}(L+\xi) \subset \mathcal{K}$ for every $(c, \xi) \in U^{\prime} \times V^{\prime}$.

Suppose by contradiction that the Mané set $\widetilde{\mathcal{N}}_{c_{0}}\left(L+\xi_{0}\right)$ is not upper-semicontinuous: There exists a neighborhood $\mathcal{V}$ of $\widetilde{\mathcal{N}}_{c_{0}}\left(L+\xi_{0}\right)$ in $T M$ such that for every neighborhood $U \times V$ of $\left(\xi_{0}, c_{0}\right)$ in $\Gamma^{\infty}(M) \times H^{1}(M ; \mathbb{R})$ we have $\tilde{\mathcal{N}}_{c}(L+\xi) \nsubseteq \mathcal{V}$ for some $(\xi, c) \in U \times V$. Then it is posssible to obtain a sequence $\left(\xi_{n}, c_{n}\right) \in U^{\prime} \times V^{\prime}$ with $\left(\xi_{n}, c_{n}\right) \rightarrow\left(\xi_{0}, c_{0}\right)$ in $\Gamma^{\infty}(M) \times H^{1}(M ; \mathbb{R})$ and $\left(x_{n}, v_{n}\right) \in \widetilde{\mathcal{N}}_{c_{n}}\left(L+\xi_{n}\right) \backslash \mathcal{V}$. Since $\tilde{\mathcal{N}}_{c_{n}}\left(L+\xi_{n}\right) \backslash \mathcal{V}$ is contained in the compact set $\mathcal{K}$ for every $n$, we can suppose the convergence $\left(x_{n}, v_{n}\right) \rightarrow\left(x_{0}, v_{0}\right) \notin \widetilde{\mathcal{N}}_{c_{0}}\left(L+\xi_{0}\right)$.

We shall prove that the Euler Lagrange solution $\left(\gamma_{n}(t), \dot{\gamma}_{n}(t)\right)=\varphi_{t}^{L+\xi_{n}-c_{n}}\left(x_{n}, v_{n}\right)$ converges on the compacts of the form $[0, T]$ :

$$
\varphi_{t}^{L+\xi_{n}-c_{n}}\left(x_{n}, v_{n}\right) \rightarrow \varphi_{t}^{L+\xi_{0}-c_{0}}\left(x_{0}, v_{0}\right) .
$$

Indeed, let $K=\sup _{(x, v) \in \mathcal{K}} L(x, v)$. So

$$
\int_{0}^{T} L\left(\gamma_{n}(t), \dot{\gamma}_{n}(t)\right) d t \leq K T
$$

Since each $\gamma_{n}$ is a $C^{k}$-curve and the actions of $L$ on the curves $\left.\gamma_{n}\right|_{[0, T]}$ are bounded by $K T$, we have that the set $\left\{\gamma_{n}\right\}$ is compact in the $C^{0}$-topology. Actually, this set is compact in the $C^{1}$-topology because we have $\left\|\dot{\gamma}_{n}\right\|$ bounded and $L_{v v}$ positive definite. Moreover, if $\gamma_{0}$ is a limit point of $\left\{\left.\gamma_{n}\right|_{[0, T]}\right\}$, so $\gamma_{0}$ is a Tonelli minimizing for the Lagrangian $L+\xi_{0}-c_{0}$. Thus if $y_{0}=\gamma_{0}(T)$ we have

$$
\begin{aligned}
\Phi_{c_{0}}^{L+\xi_{0}}\left(x_{0}, y_{0}\right) & \leq A_{c_{0}}^{L+\xi_{0}}\left(\left.\gamma_{0}\right|_{[0, T]}\right)+\alpha_{L+\xi_{0}}\left(c_{0}\right) T \\
& =\lim _{n}\left[A_{c_{n}}^{L+\xi_{n}}\left(\left.\gamma_{n}\right|_{[0, T]}\right)+\alpha_{L+\xi_{n}}\left(c_{n}\right) T\right] \\
& =\lim _{n} \Phi_{c_{n}}^{L+\xi_{n}}\left(x_{n}, \gamma_{n}(T)\right),
\end{aligned}
$$

Write $\Delta=\lim \Phi_{c_{n}}^{L+\xi_{n}}\left(x_{n}, \gamma_{n}(T)\right)$. If we prove that $\Delta=\Phi_{c_{0}}^{L+\xi_{0}}\left(x_{0}, y_{0}\right)$, then we have equality of (5):

$$
A_{c_{0}}^{L+\xi_{0}}\left(\left.\gamma_{0}\right|_{[0, T]}\right)+\alpha_{L+\xi_{0}}\left(c_{0}\right) T=\Phi_{c_{0}}^{L+\xi_{0}}\left(x_{0}, y_{0}\right)
$$

that is $\left(x_{0}, v_{0}\right) \in \widetilde{\mathcal{N}}_{c_{0}}\left(L+\xi_{0}\right)$ and we obtain a contradiction. If $\Phi_{c_{0}}^{L+\xi_{0}}\left(x_{0}, y_{0}\right)<\Delta-\varepsilon$ for some $\varepsilon>0$, then by definition of Mañé's potential, there exists a curve $\sigma:[0, S] \rightarrow$ $M$ with $\sigma(0)=x_{0}$ and $\sigma(S)=y_{0}$ such that

$$
\Phi_{c_{0}}^{L+\xi_{0}}\left(x_{0}, y_{0}\right) \leq A_{c_{0}}^{L+\xi_{0}}\left(\left.\sigma\right|_{[0, S]}\right)+\alpha_{L+\xi_{0}}\left(c_{0}\right) S<\Delta-\varepsilon .
$$


By triangular inequality property:

$$
\begin{aligned}
\Phi_{c_{n}}^{L+\xi_{n}}\left(x_{n}, \gamma_{n}(T)\right) & \leq \Phi_{c_{n}}^{L+\xi_{n}}\left(x_{n}, x_{0}\right)+\Phi_{c_{n}}^{L+\xi_{n}}\left(x_{0}, y_{0}\right)+\Phi_{c_{n}}^{L+\xi_{n}}\left(\gamma_{0}, \gamma_{n}(T)\right) \\
& \leq A_{c_{n}}^{L+\xi_{n}}\left(\left.\sigma\right|_{[0, S]}\right)+\alpha_{L+\xi_{n}}\left(c_{n}\right) S+\Phi_{c_{n}}^{L+\xi_{n}}\left(x_{n}, x_{0}\right)+\Phi_{c_{n}}^{L+\xi_{n}}\left(\gamma_{0}, \gamma_{n}(T)\right) .
\end{aligned}
$$

Given $p, q \in M$ let us take $\eta$ a geodesic with speed of norm 1 from $p$ to $q$ and $d=$ $d_{M}(p, q)$. Hence

$$
\begin{aligned}
\Phi_{c_{n}}^{L+\xi_{n}}(p, q) & \leq A_{c_{n}}^{L+\xi_{n}}\left(\left.\eta\right|_{[0, d]}\right)+\alpha_{L+\xi_{n}}\left(c_{n}\right) d \\
& =\int_{0}^{d}\left[L(\eta, \dot{\eta})+\left(\xi_{n}-c_{n}\right)(\dot{\eta})+\alpha_{L+\xi_{n}}\left(c_{n}\right)\right] d t \\
& \leq\left(\max _{\|v\|=1}|L(x, v)|+\max _{\|v\|=1}\left|\left\langle\left(\xi_{n}+c_{n}\right)(x), v\right\rangle\right|+\alpha_{L+\xi_{n}}\left(c_{n}\right)\right) d .
\end{aligned}
$$

By the continuity of critical value proved in Lemma [6, we obtain that exists $K>0$ such that for $n$ suficiently large we have $\Phi_{c_{n}}^{L+\xi_{n}}(p, q) \leq K d_{M}(p, q)$. Therefore, letting $n \rightarrow \infty$ we get $\Phi_{c_{n}}^{L+\xi_{n}}\left(x_{n}, x_{0}\right) \rightarrow 0, \Phi_{c_{n}}^{L+\xi_{n}}\left(\gamma_{0}, \gamma_{n}(T)\right) \rightarrow 0$ and, by inequality (6) $)$, we obtain a contradiction:

$$
\Delta=\lim \Phi_{c_{n}}^{L+\xi_{n}}\left(x_{n}, \gamma_{n}(T)\right) \leq A_{c_{0}}^{L+\xi_{0}}\left(\left.\sigma\right|_{[0, S]}\right)+\alpha_{L+\xi_{0}}\left(c_{0}\right) S<\Delta-\varepsilon .
$$

Now it is possible to conclude the proof of Proposition 2 stated in Introduction: Proof: (of Proposition 专) Let us take $\mathcal{U} \subset T M$ the neighborhood of $\widetilde{\mathcal{A}}_{c}(L)$ given by Lemma 4 and $B=\pi(\mathcal{U}) \subset M$ (open subset of $M$ ). Given $\varepsilon>0$, let $\lambda: M \rightarrow \mathbb{R}$ be a $C^{\infty}$ function given by

$$
\lambda(x)=\left\{\begin{array}{l}
0, \text { on } \mathcal{M}_{c}(L) \\
g(x) \text { on } B \backslash \mathcal{M}_{c}(L), \\
0 \text { on } M \backslash B
\end{array}\right.
$$

fixed a function $g$ with $0<g(x)<\varepsilon$. Let us take the $C^{\infty} 1$-form given by $\eta_{\varepsilon}(x)(v)=$ $\lambda(x)\left(\frac{\partial L}{\partial v}(x, X(x))-\frac{\partial L}{\partial v}(x, 0)\right)(v)$ where the field $X$ is given by Lemma 4 . Since $\mathfrak{M}_{c}(L)=\left\{\mu_{0}\right\}$, it follows from Lemma 5.3 in [6] that $\widetilde{\mathcal{A}}_{c}(L)=\widetilde{\mathcal{N}}_{c}(L)$. Moreover since Mañé set is upper-semicontinuous with respect to 1-forms (Proposition 5), for $\varepsilon$ sufficiently small, taking $\eta=\eta_{\varepsilon}$ we obtain $\widetilde{\mathcal{N}}_{c}\left(L+\eta_{\varepsilon}\right) \subset \mathcal{U}$. Hence

$$
\widetilde{\mathcal{M}}_{c}(L+\eta) \subset \widetilde{\mathcal{A}}_{c}(L+\eta) \subset \widetilde{\mathcal{N}}_{c}(L+\eta) \subset \mathcal{U} .
$$

This means that for every $\mu \in \mathfrak{M}_{c}(L+\eta)$ we have $\operatorname{supp}(\mu) \subset \mathcal{U}$ and by Lemma 4 we obtain

$$
\int_{T M} \eta d \mu=\int_{T M} \eta(x) v d \mu \geq 0 .
$$


Let us to show that

$$
\widetilde{\mathcal{M}}_{c}(L+\eta)=\widetilde{\mathcal{M}}_{c}(L)
$$

Indeed, since $\left.\eta\right|_{\widetilde{\mathcal{M}}_{c}(L)} \equiv 0$, we have

$$
A_{c}^{L+\eta}\left(\mu_{0}\right)=A_{c}^{L}\left(\mu_{0}\right) \leq A_{c}^{L}(\mu) \leq A_{c}^{L+\eta}(\mu), \forall \mu \in \mathfrak{M}_{c}(L+\eta),
$$

That is

$$
\mu_{0} \in \mathfrak{M}_{c}(L+\eta) .
$$

On the other hand, if $\delta \in \mathfrak{M}_{c}(L+\eta)$,

$$
A_{c}^{L}(\delta) \leq A_{c}^{L+\eta}(\delta)=A_{c}^{L+\eta}\left(\mu_{0}\right)=A_{c}^{L}\left(\mu_{0}\right),
$$

Therefore $\delta \in \mathfrak{M}_{c}(L)$ and we conclude that

$$
\widetilde{\mathcal{M}}_{c}(L+\eta)=\operatorname{supp}\left(\mu_{0}\right) \text {. }
$$

Let us suppose that $\widetilde{\mathcal{A}}_{c}(L+\eta) \neq \widetilde{\mathcal{M}}_{c}(L+\eta)$. Recall that since the Graph Property holds for $\widetilde{\mathcal{A}}_{c}(L+\eta)$, there exists

$$
x \in \mathcal{A}_{c}(L+\eta) \backslash \mathcal{M}_{c}(L+\eta) .
$$

Let $\gamma: \mathbb{R} \rightarrow M$ be the minimizing curve for $L+\eta-c$ with $\gamma(0)=x$. It follows from 8 that $(\gamma, \dot{\gamma})(\mathbb{R}) \subset \mathcal{U}$. Since $\mu_{0}$ is ergodic, almost every point has a dense orbit on $\operatorname{supp}\left(\mu_{0}\right)$. Let $z \in \operatorname{supp}\left(\mu_{0}\right)$ be such that it has a dense orbit $(\sigma, \dot{\sigma})$ on $\operatorname{supp}\left(\mu_{0}\right)$. Then given $u, w \in \pi\left(\operatorname{supp}\left(\mu_{0}\right)\right)$, we can take $t_{n}>s_{n}>0$ such that $u=\lim _{n} \sigma\left(s_{n}\right)$ and $w=\lim _{n} \sigma\left(t_{n}\right)$. Observe that the critical values $\alpha_{L}(c)=\alpha_{L+\eta}(c)=\alpha$ are the same. Moreover as we mentioned before $\left.\eta\right|_{\operatorname{supp}\left(\mu_{0}\right)} \equiv 0$. Hence

$$
\begin{aligned}
\Phi_{c}^{L+\eta}(u, w) & =\lim _{n} \Phi_{c}^{L+\eta}\left(\sigma\left(s_{n}\right), \sigma\left(t_{n}\right)\right)=\lim _{n} A_{c}^{L+\eta+\alpha}\left(\left.\sigma\right|_{\left[s_{n}, t_{n}\right]}\right) \\
& =\lim _{n} A_{c}^{L+\alpha}\left(\left.\sigma\right|_{\left[s_{n}, t_{n}\right]}\right)=\lim _{n} \Phi_{c}^{L}\left(\sigma\left(s_{n}\right), \sigma\left(t_{n}\right)\right) \\
& =\Phi_{c}^{L}(u, w) .
\end{aligned}
$$

It is known that for every $(y, w) \in \widetilde{\mathcal{N}}_{c}(L+\eta)$, the $\omega$ and $\alpha$-limit sets of $(y, w)$ are contained in $\operatorname{supp}\left(\mu_{0}\right)$ (because $\left.\widetilde{\mathcal{M}}_{c}(L+\eta)=\operatorname{supp}\left(\mu_{0}\right)\right)$. Hence $\omega$ and $\alpha$-limits of $x$ are contained in $\pi\left(\operatorname{supp}\left(\mu_{0}\right)\right)$. Now let $t_{n}, s_{n} \rightarrow \infty$ such that $u_{0}=\lim _{n} \gamma\left(t_{n}\right)$ and $w_{0}=\lim _{n} \gamma\left(-s_{n}\right)$. We observe that $x \notin \mathcal{M}_{c}(L)=\mathcal{M}_{c}(L+\eta)$ then for $n_{0}$ sufficiently big, $\left.\gamma\right|_{\left[t_{n_{0}}, t_{n}\right]} \subset B \backslash \mathcal{M}_{c}(L)$ for every $t_{n}>t_{n_{0}}$. By the definition of $\lambda$, there exists $a>0$ such that $\int_{-s_{n}}^{t_{n}} \lambda(\gamma(t)) d t>a$. Therefore by Lemma 4 we have

$$
\int_{-s_{n}}^{t_{n}} \eta(\gamma(t)) \dot{\gamma}(t) d t \geq \int_{-s_{n}}^{t_{n}} \lambda(\gamma(t)) K d t>a K .
$$


Hence

$$
\begin{aligned}
0 & =\Phi_{c}^{L+\eta}\left(\gamma\left(-s_{n}\right), \gamma\left(t_{n}\right)\right)+\Phi_{c}^{L+\eta}\left(\gamma\left(t_{n}\right), \gamma\left(-s_{n}\right)\right) \\
& =A_{c}^{L+\eta+\alpha}\left(\left.\gamma\right|_{\left[-s_{n}, t_{n}\right]}\right)+\Phi_{c}^{L+\eta}\left(\gamma\left(t_{n}\right), \gamma\left(-s_{n}\right)\right) \\
& >A_{c}^{L+\alpha}\left(\left.\gamma\right|_{\left[-s_{n}, t_{n}\right]}\right)+a K+\Phi_{c}^{L+\eta}\left(\gamma\left(t_{n}\right), \gamma\left(-s_{n}\right)\right) \\
& \geq \Phi_{c}^{L}\left(\gamma\left(-s_{n}\right), \gamma\left(t_{n}\right)\right)+\Phi_{c}^{L+\eta}\left(\gamma\left(t_{n}\right), \gamma\left(-s_{n}\right)\right)+a K .
\end{aligned}
$$

Taking limit as $n \rightarrow \infty$ we obtain

$$
\begin{aligned}
0 & \geq \Phi_{c}^{L}\left(w_{0}, u_{0}\right)+\Phi_{c}^{L+\eta}\left(u_{0}, w_{0}\right)+a K \\
& =\Phi_{c}^{L}\left(w_{0}, u_{0}\right)+\Phi_{c}^{L}\left(u_{0}, w_{0}\right)+a K \geq a K .
\end{aligned}
$$

This contradiction implies that

$$
\widetilde{\mathcal{A}}_{c}(L+\eta)=\widetilde{\mathcal{M}}_{c}(L+\eta)=\widetilde{\mathcal{M}}_{c}(L)
$$

Since $\# \mathfrak{M}_{c}(L+\eta)=1$ it follows from Lemma 5.3 in [6] that $\widetilde{\mathcal{A}}_{c}(L+\eta)=$ $\widetilde{\mathcal{N}}_{c}(L+\eta)$. Then we conclude the proof.

\subsection{The exact magnetic Lagrangian case}

Now let $L$ be an exact magnetic Lagrangian. That is

$$
L(x, v)=\frac{\|v\|^{2}}{2}+\xi_{x}(v)
$$

for some non-closed 1 -form $\xi_{x}$. We will prove that $\widetilde{\mathcal{M}}_{c}(L)$ supports on a uniquely ergodic measure for a residual set of $H^{1}(M ; \mathbb{R})$. In order for this, we need of following conclusion derived from Theorem 1.1 in [2]:

Theorem 7 Let $L$ be an exact magnetic Lagrangian. Given a cohomology class c, there exists a residual subset $\mathcal{O}_{c}$ of $\Gamma^{\infty}(M)$ such that for any $\omega \in \mathcal{O}_{c}, \widetilde{\mathcal{M}}_{c}(L+\omega)$ supports on a uniquely ergodic measure.

Since the subset $\Lambda \subset \Gamma^{\infty}(M)$ of non-closed 1-forms is open and dense in $\Gamma^{\infty}(M)$, we can consider the residual $\mathcal{O}_{c}$, intercepting with $\Lambda$ if necessary, such that its elements are non-closed 1-forms and such that $\xi_{x}+\omega$ are non-closed 1-forms. This means that $L+\omega$ is also an exact magnetic Lagrangian. In this case, the magnetic field of the perturbed Lagrangian changes the Lorentz force. 
Observe that the 1-form obtained in Proposition 2 is given by

$$
\eta_{x}(v)=\lambda(x)\left(\frac{\partial L}{\partial v}(x, X(x))-\frac{\partial L}{\partial v}(x, 0)\right)(v),
$$

where the $C^{\infty}$ function $\lambda: M \rightarrow \mathbb{R}$ is given in 7 and the field $X$ is given by Lemma 4 , Then for the exact magnetic Lagrangian case, we have $\eta_{x}(v)=\langle\lambda(x) X(x), v\rangle$.

With these notations we obtain the following corollary:

Corollary 8 Let $L$ be an exact magnetic Lagrangian. Given a cohomology class $c \in$ $H^{1}(M ; \mathbb{R})$ and a 1-form $\omega \in \mathcal{O}_{c}$, there exists a $C^{\infty} 1$-form $\eta$ (sufficiently close to zero) such that

$$
\widetilde{\mathcal{M}}_{c}(L+\omega+\eta)=\widetilde{\mathcal{A}}_{c}(L+\omega+\eta)=\widetilde{\mathcal{N}}_{c}(L+\omega+\eta)=\widetilde{\mathcal{M}}_{c}(L+\omega),
$$

with $\widetilde{\mathcal{M}}_{c}(L+\omega)$ supports on a uniquely ergodic measure.

Proof: It follows from [11], Corollary 5.1 that $\alpha(c)>e_{0}$ for every $c \in H^{1}(M ; \mathbb{R})$. Then the proof follows directly from Proposition 2 and remarks above.

In order to state some direct consequences from Proposition 2 , let us take $\zeta=$ $\left\{c_{n}\right\}_{n=1}^{\infty}$ a dense sequence in $H^{1}(M ; \mathbb{R})$.

Corollary 9 Let $L$ be an exact magnetic Lagrangian. Then there exists a residual subset $\mathcal{O}^{\prime}$ of $\Gamma^{\infty}(M)$ such that for each $\omega \in \mathcal{O}^{\prime}$ and each $c_{n} \in \zeta$, there exists a 1-form $\eta_{\omega, n}$ (sufficiently close to 0 ) such that

$$
\widetilde{\mathcal{M}}_{c_{n}}\left(L+\omega+\eta_{\omega, n}\right)=\widetilde{\mathcal{A}}_{c_{n}}\left(L+\omega+\eta_{\omega, n}\right)=\widetilde{\mathcal{N}}_{c_{n}}\left(L+\omega+\eta_{\omega, n}\right)
$$

with $\widetilde{\mathcal{M}}_{c_{n}}\left(L+\omega+\eta_{\omega, n}\right)$ supports on a uniquely ergodic measure.

Proof: It follows from Theorem 7 that there exists a residual subset $\mathcal{O}_{c_{n}}$ of $\Gamma^{\infty}(M)$ such that for any $\omega \in \mathcal{O}_{c_{n}}, \widetilde{\mathcal{M}}_{c_{n}}(L+\omega)$ supports on a uniquely ergodic measure. Let $\mathcal{O}^{\prime}$ be the residual subset $\mathcal{O}^{\prime}=\cap \mathcal{O}_{c_{n}}$ of $\Gamma^{\infty}(M)$. Now, for each $\omega \in \mathcal{O}^{\prime}$, by taking $L+\omega$ and $c=c_{n}$ in Proposition Q we conclude that there exists $\eta_{\omega, n}$ sufficiently close to 0 that satisfies the statement.

Corollary 10 Let $L$ be an exact magnetic Lagrangian. Then for each $c_{n} \in \zeta$ there exists a dense set $\mathcal{O}_{n}$ of $\Gamma^{\infty}(M)$ such that

$$
\widetilde{\mathcal{M}}_{c_{n}}\left(L+\varphi_{n}\right)=\widetilde{\mathcal{A}}_{c_{n}}\left(L+\varphi_{n}\right)=\widetilde{\mathcal{N}}_{c_{n}}\left(L+\varphi_{n}\right)
$$

and $\widetilde{\mathcal{M}}_{c_{n}}\left(L+\varphi_{n}\right)$ supports on a uniquely ergodic measure, for every $\varphi_{n} \in \mathcal{O}_{n}$. 
Proof: Let $\mathcal{O}^{\prime}$ be the residual subset obtained from Corollary 9. We can vary $\omega \in$ $\mathcal{O}^{\prime}$ (which is dense in $\left.\Gamma^{\infty}(M)\right)$ in order to obtain the following dense set

$$
\mathcal{O}_{n}=\left\{\varphi_{n}=\omega+\eta_{\omega, n}: \omega \in \mathcal{O}^{\prime}\right\}
$$

for each $n \in \mathbb{N}$. This proves the statement.

Finally, we can prove Theorem 1 ;

Proof: (of Theorem 11) Fix $c_{n} \in \zeta$ and $\sigma=\varphi_{n} \in \mathcal{O}_{n}$ given by Corollary 10, Consider the following neighborhood of $\widetilde{\mathcal{M}}_{c_{n}}(L+\sigma)$ in $T M$ :

$$
\mathcal{V}_{n, r}(\sigma)=\left\{P \in T M: d_{T M}\left(P, \widetilde{\mathcal{M}}_{c_{n}}(L+\sigma)\right)<\frac{1}{r}\right\},
$$

Given $\varepsilon>0$, let $\left\{z_{1}, \ldots, z_{N}\right\} \subset \widetilde{\mathcal{M}}_{c_{n}}(L+\sigma)=\operatorname{supp}\left(\mu_{n}\right)$ be such that $\operatorname{supp}\left(\mu_{n}\right) \subset$ $\bigcup_{i=1}^{N} B\left(z_{i}, \frac{1}{r}\right)$, where $B\left(z_{i}, \frac{1}{r}\right) \subset \mathcal{V}_{n, r}(\sigma)$ is the open ball of center $z_{i}$ and radius $\frac{1}{r}$ in $T M$. There exists a open $A_{n, r}(\sigma)$ of $\left(\sigma, c_{n}\right)$ in $\Gamma^{\infty}(M) \times H^{1}(M ; \mathbb{R})$ such that for every $(\xi, c) \in A_{n, r}(\sigma)$ we have

$$
\sup _{P \in \widetilde{\mathcal{M}}_{c_{n}}(L+\sigma)} d_{T M}\left(P, \widetilde{\mathcal{M}}_{c}(L+\xi)\right)<\frac{1}{r}
$$

Indeed, otherwise, we obtain sequences $c_{n}^{k} \rightarrow c_{n}, \xi_{k} \rightarrow \sigma($ as $k \rightarrow \infty)$ and $P_{k} \in$ $\widetilde{\mathcal{M}}_{c_{n}}(L+\sigma)=\operatorname{supp}\left(\mu_{n}\right)$ such that $d_{T M}\left(P_{k}, \widetilde{\mathcal{M}}_{c_{n}^{k}}\left(L+\xi_{k}\right)\right) \geq \frac{1}{r}$. We consider a sequence of minimizing measures $\mu_{n}^{k}, \operatorname{supp}\left(\mu_{n}^{k}\right) \subset \widetilde{\mathcal{M}}_{c}(L+\xi)$. The continuity of $\alpha_{L+\xi}(c)$ implies that $\mu_{n}^{k} \rightarrow \mu_{n}$ (as $\left.k \rightarrow \infty\right)$ weakly*. Hence if $g_{i}: T M \rightarrow \mathbb{R}$ is a positive continuous function with

$$
B\left(z_{i}, \frac{1}{r}\right) \cap \operatorname{supp}\left(\mu_{n}\right) \subset \operatorname{supp}\left(g_{i}\right) \subset B\left(z_{i}, \frac{1}{r}\right),
$$

we have $\int g_{i} d \mu_{n}>0$ for every $i=1, \ldots, N$. Then $\int g_{i} d \mu_{n}^{k}>0$, for $k$ sufficiently big and for any $i=1, \ldots, N$, that implies $B\left(z_{i}, \frac{1}{r}\right) \cap \operatorname{supp}\left(\mu_{n}^{k}\right) \neq \emptyset$. Since $P_{k} \in B\left(z_{i}, \frac{1}{r}\right)$ for some $i=1, \ldots, N$ we obtain $d_{T M}\left(P_{k}, \operatorname{supp}\left(\mu_{n}^{k}\right)\right)<\frac{1}{r}$. This is an absurd.

As the map $(\xi, c) \in \Gamma^{\infty}(M) \times H^{1}(M ; \mathbb{R}) \longmapsto \widetilde{\mathcal{N}}_{c}(L+\xi)$ is upper-semicontinuous and $\widetilde{\mathcal{M}}_{c_{n}}(L+\sigma)=\widetilde{\mathcal{N}}_{c_{n}}(L+\sigma)$ for every $\sigma \in \mathcal{O}_{n}$, we can consider the neighborhood $A_{n, r}(\sigma)$ of $\left(\sigma, c_{n}\right)$ in $\Gamma^{\infty}(M) \times H^{1}(M ; \mathbb{R})$ such that $\widetilde{\mathcal{N}}_{c}(L+\xi) \subset \mathcal{V}_{n, r}(\sigma)$ for every $(\xi, c) \in A_{n, r}(\sigma)$ and for each $\sigma \in \mathcal{O}_{n}$. Let us take the subset open $B_{n, r}=\bigcup_{\sigma \in \mathcal{O}_{n}} A_{n, r}(\sigma) \subset$ $\Gamma^{\infty}(M) \times H^{1}(M ; \mathbb{R}), U_{n, r}=\pi_{1}\left(B_{n, r}\right)$ and $V_{n, r}=\pi_{2}\left(B_{n, r}\right)$. Hence $U_{n, r} \supset \mathcal{O}_{n}$ and

$$
\widetilde{\mathcal{M}}_{c}(L+\xi) \subset \widetilde{\mathcal{A}}_{c}(L+\xi) \subset \widetilde{\mathcal{N}}_{c}(L+\xi) \subset \mathcal{V}_{n, r}(\sigma), \forall(\xi, c) \in U_{n, r} \times V_{n, r}
$$


Let us take $\mathcal{O}^{\prime \prime}=\bigcap_{n, r} U_{n, r}, \mathcal{O}=\mathcal{O}^{\prime} \cap \mathcal{O}^{\prime \prime}$ (given by Corollary 9) and $\mathcal{G}=\bigcap_{r}\left(\bigcup_{n} V_{n, r}\right)$. Observe that $\bigcup_{n} V_{n, r}$ is a open and dense subset of $H^{1}(M ; \mathbb{R})$.

Now let us to show that

$$
\widetilde{\mathcal{M}}_{c}(L+\xi)=\widetilde{\mathcal{A}}_{c}(L+\xi)=\widetilde{\mathcal{N}}_{c}(L+\xi), \forall(\xi, c) \in \mathcal{O} \times \mathcal{G}
$$

Indeed, let us take $R \in \widetilde{\mathcal{N}}_{c}(L+\xi)$ and an integer $k>0$. As $c \in \mathcal{G}$ we can find $m \in \mathbb{N}$ such that $c \in V_{m, 2 k}$ (Neighborhood of $c_{m}$ ). Since $\xi \in \mathcal{O}$ we have $\xi \in U_{m, 2 k}$. Hence $(\xi, c) \in B_{m, 2 k}$ and there exists $\sigma_{m} \in \mathcal{O}_{m}$ such that $(\xi, c) \in A_{m, 2 k}\left(\sigma_{m}\right)$, that is

$$
\widetilde{\mathcal{M}}_{c}(L+\xi) \subset \widetilde{\mathcal{A}}_{c}(L+\xi) \subset \widetilde{\mathcal{N}}_{c}(L+\xi) \subset \mathcal{V}_{m, 2 k}\left(\sigma_{m}\right)
$$

Let $Q \in \widetilde{\mathcal{M}}_{c_{m}}\left(L+\sigma_{m}\right)$ be the minimum point:

$$
d_{T M}\left(R, \widetilde{\mathcal{M}}_{c_{m}}\left(L+\sigma_{m}\right)\right)=d_{T M}(R, Q),
$$

and $S \in \widetilde{\mathcal{M}}_{c}(L+\xi)$ the minimum point:

$$
d_{T M}\left(Q, \widetilde{\mathcal{M}}_{c}(L+\xi)\right)=d_{T M}(Q, S)
$$

It follows from 10 and 11 that

$$
\begin{aligned}
d_{T M}\left(R, \widetilde{\mathcal{M}}_{c}(L+\xi)\right) & \leq d_{T M}(R, S) \leq d_{T M}(R, Q)+d_{T M}(Q, S) \\
& =d_{T M}\left(R, \widetilde{\mathcal{M}}_{c_{m}}\left(L+\sigma_{m}\right)\right)+d_{T M}\left(Q, \widetilde{\mathcal{M}}_{c}(L+\xi)\right) \\
& <\frac{1}{2 k}+\frac{1}{2 k}=\frac{1}{k} .
\end{aligned}
$$

Since this holds for any $k>0$ and $\widetilde{\mathcal{M}}_{c}(L+\xi)$ is compact, we conclude that $R \in$ $\widetilde{\mathcal{M}}_{c}(L+\xi)$.

\section{Hyperbolic periodic orbit for a perturbed exact magnetic Lagrangian}

The main goal of this section is to prove Theorem 3. Before we must prove the following proposition that holds for Tonelli Lagrangians. The idea of the proof is to use the results obtained by $\mathrm{G}$. Contreras and R. Iturriaga in [4] on the index forms. 
Let $\Omega_{T}$ be the set of continuous piecewise $C^{2}$ vectorfields $\xi$ along a curve $\left.\gamma\right|_{[0, T]}$. The index form on $\Omega_{T}$ is defined by

$$
I(\xi, \zeta)=\int_{0}^{T}\left(L_{v v}(\dot{\xi}, \dot{\zeta})+L_{v x}(\dot{\xi}, \zeta)+L_{x v}(\xi, \dot{\zeta})+L_{v v}(\xi, \zeta)\right) d t
$$

For more details on this form see [4], Section 4.

Proposition 11 Let $L$ be a Tonelli Lagrangian and $c$ be a cohomology class with $\alpha(c)>e_{0}$. Let us suppose that $\widetilde{\mathcal{M}}_{c}(L)$ has a unique minimizing measure supported on a periodic orbit. Then there exists a $C^{\infty}$ 1-form $\eta$ (sufficiently close to zero) such that the perturbed Lagrangian $L+\eta-c$ has a unique minimizing measure supported on a hyperbolic periodic orbit $\Gamma$. Moreover the stable and unstable manifolds of $\Gamma$ intersect transversally $W^{s}(\Gamma) \pitchfork W^{u}(\Gamma)$.

Proof: We can consider $c=0$. Let $\Gamma$ the minimizing periodic orbit in $\widetilde{\mathcal{M}}(L)$. By the graph property, $\left.\pi\right|_{\Gamma}: \Gamma \rightarrow M, \pi(x, v)=x$ is injective, so $\pi(\Gamma) \subset M$ is a simple closed curve. We consider coordinates on a tubular neighborhood of $\pi(\Gamma)$ in the following way: $\varphi: U \rightarrow S^{1} \times \mathbb{R}^{n-1}, \varphi=\varphi\left(x_{1}, \ldots, x_{n}\right)$ with $\varphi(\Gamma)=S^{1} \times\{0\}$ and $\left\{\frac{\partial}{\partial x_{1}}, \frac{\partial}{\partial x_{2}}, \ldots, \frac{\partial}{\partial x_{n}}\right\}$ is an orthonormal frame over the points of $\pi(\Gamma)=\gamma$.

Given $\varepsilon>0$,we take the $C^{\infty}$ function $\lambda: M \rightarrow \mathbb{R}$, given by 7 in the proof of Proposition 2, as

$$
\lambda(x)=\left\{\begin{array}{l}
0, \text { on } \pi(\Gamma) \\
\frac{\varepsilon}{2} f(x)\left(x_{2}^{2}+x_{3}^{2}+\ldots+x_{n}^{2}\right) \text { on } B \backslash \pi(\Gamma), \\
0 \text { on } M \backslash B
\end{array}\right.
$$

where $B \subset U$ and $f$ is a non-negative bump function with support contained in $B$ and which is one on a small neighborhood of $\pi(\Gamma)$. Then by Proposition 0 and Equation 9. the 1-form

$$
\eta(x, v)=\eta_{x}(v)=\lambda(x)\left(L_{v}(x, X(x))-L_{v}(x, 0)\right)(v)
$$

is such that

$$
\widetilde{\mathcal{M}}(L+\eta)=\widetilde{\mathcal{M}}(L)=\Gamma .
$$

Note that $\eta$ can be made $C^{\infty}$ arbitrarily small. Now we define $\tilde{L}=L+\eta$. In order to aplly the index form [12, let us calculate the derivatives $\tilde{L}_{v v}, \tilde{L}_{v x}$ and $\tilde{L}_{x x}$ on $\Gamma$ : we have $\tilde{L}_{v v}=L_{v v}$ and since $\partial_{v} \eta(x, v)(h)=\eta_{x}(h)=\eta(x, h) \forall h \in T_{x} M$, we conclude that

$$
\tilde{L}_{v x}(h, k)=L_{v x}(h, k)+\left[\partial_{x} \eta(x, h)\right](k) .
$$


By taking the 1-form

$$
\omega(x, v)=\omega_{x}(v)=\left(L_{v}(x, X(x))-L_{v}(x, 0)\right)(v),
$$

we have $\eta_{x}=\lambda(x) \omega_{x}$. Then

$$
\left[\partial_{x} \eta(x, h)\right](k)=d \lambda(x)(k) \omega(x, h)+\lambda(x)\left[\partial_{x} \omega(x, h)\right](k) .
$$

In coordinates we have

$$
d_{x} \lambda(x)(k)=\frac{1}{2} \varepsilon\left(x_{2}^{2}+x_{3}^{2}+\ldots+x_{n}^{2}\right)\left[d_{x} f(x)(k)\right]+f(x) \varepsilon\left(0, x_{2}, x_{3}, \ldots, x_{n}\right)(k) .
$$

Since $\left.\lambda\right|_{\pi(\Gamma)}=0$ we conclude that $\left.d_{x} \lambda\right|_{\pi(\Gamma)}=0$ and $\left.\partial_{x} \eta\right|_{\pi(\Gamma)}=0$. Therefore $\tilde{L}_{v x}=L_{v x}$ on $\Gamma$.

It ramains to calculate $\tilde{L}_{x x}$. Observe that

$$
\begin{aligned}
\tilde{L}_{x x}(x, v)(h, k)= & L_{x x}(x, v)(h, k)+\partial_{x x} \eta(x, v)(h, k) \\
= & L_{x x}(x, v)(h, k)+d_{x}^{2} \lambda(x)(h, k) \omega(x, v)+d_{x} \lambda(x)(h) \partial_{x} \omega(x, v)(k) \\
& +d_{x} \lambda(x)(k) \partial_{x} \omega(x, v)(h)+\lambda(x) \partial_{x x} \omega(x, v)(h, k) .
\end{aligned}
$$

Hence,

$$
\tilde{L}_{x x}(x, v)(h, k)=L_{x x}(h, k)+d_{x}^{2} \lambda(x)(h, k) \omega(x, v) \text { on } \pi(\Gamma) .
$$

Now it follows from 14 that

$$
d_{x}^{2} \lambda(x)(h, k)=f(x) \varepsilon\left[\begin{array}{ll}
0 & 0 \\
0 & I
\end{array}\right](h, k) \text { on } \pi(\Gamma),
$$

where $I$ is the identity matrix $(n-1) \times(n-1)$.

Therefore, on $\Gamma$, we have $\tilde{L}_{v v}=L_{v v}, \tilde{L}_{v x}=L_{v x}$ and

$$
\tilde{L}_{x x}(x, v)(h, k)=L_{x x}(h, k)+f(x) \varepsilon\left[\begin{array}{ll}
0 & 0 \\
0 & I
\end{array}\right](h, k)\left(L_{v}(x, X(x))-L_{v}(x, 0)\right)(v) .
$$

Now it is possible to compare the index of the original and the perturbed lagrangian along the solution. Let $\tilde{I}_{T}$ and $I_{T}$ be the index forms on $[0, T]$ for $\tilde{L}$ and $L$, respectvely. Fix $\theta \in \Gamma$ and define $N(\theta)=\left\{w \in T_{\pi(\theta)} M \mid\langle w, \dot{\gamma}\rangle=0\right\}$. Hence $N(\theta)$ is generated by the vectors $\frac{\partial}{\partial x_{2}}, \ldots, \frac{\partial}{\partial x_{n}}$. Denote $\pi_{N}\left(\xi_{1}, \xi_{2}, \ldots, \xi_{n}\right)=\left(\xi_{2}, \ldots, \xi_{n}\right)$. Since the next steps of proof hold for Tonelli Lagrangians in general, even in our case, they are entirely analogous to proof of Theorem D in [4] (Section 5, page 934). Therefore it is suffices to proof that there exists $\delta>0$, such that $\tilde{I}_{T}\left(\xi^{T}, \xi^{T}\right) \geq I_{T}\left(\xi^{T}, \xi^{T}\right)+\delta$, for certain 
vectors $\xi^{T}$ satisfying $\left|\pi_{N}\left(\xi^{T}(t)\right)\right|>\frac{1}{2}$ for every $0 \leq t \leq \lambda$ and $T>T_{0}$. Indeed, in the coordinates $\left(x_{1}, \ldots, x_{n}, \frac{\partial}{\partial x_{2}}, \ldots, \frac{\partial}{\partial x_{n}}\right)$ on $T U$ we have that

$$
\begin{aligned}
\tilde{I}_{T}\left(\xi^{T}, \xi^{T}\right)= & \int_{0}^{T}\left(\tilde{L}_{v v}\left(\dot{\xi}^{T}, \dot{\xi}^{T}\right)+2 \tilde{L}_{v x}\left(\dot{\xi}^{T}, \xi^{T}\right)+\tilde{L}_{x x}\left(\xi^{T}, \xi^{T}\right)\right) d t \\
= & \int_{0}^{T}\left(L_{v v}\left(\dot{\xi}^{T}, \dot{\xi}^{T}\right)+2 L_{v x}\left(\dot{\xi}^{T}, \xi^{T}\right)+L_{x x}\left(\xi^{T}, \xi^{T}\right)\right) d t \\
& +\int_{0}^{T}\left(f(\gamma) \varepsilon\left[\begin{array}{cc}
0 & 0 \\
0 & I
\end{array}\right]\left(\xi^{T}, \xi^{T}\right)\left(L_{v}(\gamma, X(\gamma))-L_{v}(\gamma, 0)\right)(\dot{\gamma})\right) d t \\
\geq & I_{T}\left(\xi^{T}, \xi^{T}\right)+\int_{0}^{T} \varepsilon \sum_{i=2}^{n}\left(\xi_{i}^{T}\right)^{2} K d t \geq I_{T}\left(\xi^{T}, \xi^{T}\right)+\varepsilon \frac{K \lambda}{4},
\end{aligned}
$$

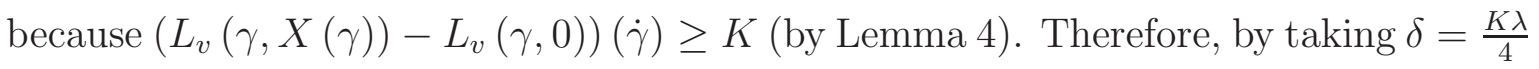
we obtain that $\Gamma$ is a hyperbolic periodic orbit for the Lagrangian $L+\eta$. Now we must prove by perturbing $L+\eta$, if necessary, that the stable and unstable manifolds intersect transversally $W^{s}(\Gamma) \pitchfork W^{u}(\Gamma)$. Actually, using similar steps as above, the proof follows from the same arguments as in the proof of Theorem D in [4] (Section 5, page 934).

Finally we can conclude the proof of Theorem 3 stated in Introduction.

Proof: (of Theorem (3) We aplly Theorem 7 for $c=0$ to deduce that there exists a residual subset $\mathcal{O}$ of $\Gamma^{\infty}(M)$ such that for any $\omega \in \mathcal{O}$, the Lagrangian $L+\omega$ has a unique minimizing measure and this measure is uniquely ergodic. Let $\mathcal{A}$ be the subset of $\mathcal{O}$ of 1 -forms for which the measure on $\mathfrak{M}(L+\omega)$ is supported on a periodic orbit. Let $\mathcal{A}_{1}$ be the subset of $\mathcal{A}$ on which the minimizing periodic orbit is hyperbolic and its stable and unstable manifolds intersect transversally $W^{s}(\Gamma) \pitchfork W^{u}(\Gamma)$. The proof that $\mathcal{A}_{1}$ is relatively open on $\mathcal{A}$ and the final step are entirely analogous to proof of Theorem $\mathrm{D}$ in [4]. We repeat the final step here only for the sake of completeness.

Let $\mathcal{U}$ be an open subset of $\Gamma^{\infty}(M)$ such that $\mathcal{A}_{1}=\mathcal{A} \cap \mathcal{U}$. Let $\mathcal{B}:=\mathcal{O} \backslash \mathcal{A}$. Since for an exact magnetic Lagrangian we have $\alpha(c)>e_{0}$ for every cohomology class $c$ (see [11], Corollary 5.1), we can use Proposition 11 to conclude that $\mathcal{A}_{1}$ is dense in $\mathcal{A}$. Therefore $\mathcal{A}_{1} \cup \mathcal{B}$ is generic in $\Gamma^{\infty}(M)$. Let $\mathcal{V}=\operatorname{int}\left(\Gamma^{\infty}(M) \backslash \mathcal{U}\right)$. Hence $\mathcal{U} \cup \mathcal{V}$ is an open and dense in $\Gamma^{\infty}(M)$. Since $\mathcal{A} \subset \overline{\mathcal{A}_{1}} \subset \overline{\mathcal{U}}$ we have $\mathcal{A} \cap \mathcal{V} \subset \overline{\mathcal{U}} \cap \mathcal{V}=\emptyset$, that is $\mathcal{A} \cap \mathcal{V}=\emptyset$. Moreover $\mathcal{O}=\mathcal{A} \cup \mathcal{B}$ is generic and

$$
\begin{aligned}
(\mathcal{U} \cup \mathcal{V}) \cap(\mathcal{A} \cup \mathcal{B}) & =[(\mathcal{U} \cup \mathcal{V}) \cap \mathcal{A}] \cup[(\mathcal{U} \cup \mathcal{V}) \cap \mathcal{B}] \\
& =(\mathcal{U} \cap \mathcal{A}) \cup[(\mathcal{U} \cup \mathcal{V}) \cap \mathcal{B}] \\
& \subset \mathcal{A}_{1} \cup \mathcal{B}
\end{aligned}
$$

This shows that $\mathcal{A}_{1} \cup \mathcal{B}$ is generic in $\Gamma^{\infty}(M)$. 


\section{Acknowledgements}

I am grateful to Mário Jorge Dias Carneiro and José Antônio Gonçalves Miranda for several helpful comments and suggestions. I thank also to FAPEMIG-BRAZIL which supported partially this work.

\section{References}

[1] Arbieto, A., Castro, F., Generic properties of magnetic flows, arXiv:1601.00935 (2016)

[2] Carneiro, M. J., Rocha, A., A generic property of exact magnetic Lagrangians, Discrete and Continuous Dynamical Systems, 32, N.12 (2012).

[3] Contreras, G., Delgado J., Iturriaga R., Lagrangian flows: the dynamics of globally minimizing orbits II, Bol. Soc. Bras. Mat. 28 (2) 155-196, (1997).

[4] Contreras, G., Iturriaga, R., Convex Hamiltonians without conjugate points, Ergodic Theory Dynam. Systems, 19, 901-952 (1999).

[5] Contreras, G., Iturriaga, R., Global Minimizers of Autonomous Lagrangians, 22 Colóquio Brasileiro de Matemática, (1999).

[6] Contreras, G., Paternain, P., Connecting orbits between static classes for generic Lagrangian systems, Topology, 41 645-666, (2002).

[7] Miranda, J. A. G., Generic properties for magnetic flows on surfaces, Nonlinearity 19, 1849-1874, (2006).

[8] Mañé, R., Generic properties and problems of minimizing measure of Lagrangian dynamical systems, Nonlinearity, 9, N.2, 273-310, (1996).

[9] Mañé, R., Lagrangian flows: the dynamics of globally minimizing orbits. In International Conference on Dynamical Systems (Montevideo, 1995). Longman, Harlow, 1996, pp. 120-131. Reprinted in Bol. Soc.Brasil. Mat. (N.S.) 28(2), 141-153 (1997).

[10] Mather, J. N., Action minimizing invariant measures for positive definite Lagrangian Systems, Math. Zeitschrift, 207, 169-207, (1991). 
[11] Paternain, M., Paternain, G., Critical Values of autonomous Lagrangian systems, Comment. Math. Helvetici, 7 481, (1997).

[12] Zhang, J., Generically Mañé set supports uniquely ergodic measure for residual cohomology class, Proceedings of the American Mathematical Society 145(9), (2016). 